\title{
A Study for New Balance Sports Products to Analyze and Design
}

\author{
Yu-Che Huang ${ }^{1}$, Tai-Shen Huang ${ }^{1}$ \\ ${ }^{1} 168$, Jifeng E. Rd., Wufeng District, Taichung, 41349, Taiwan
}

\begin{abstract}
In recent years, under the influence of globalization, more and more different needs have created different sports products. In general, they are mainly used for cardio training and muscular endurance training, and their products for human balance training are few. Balance training has always been one of the most important exercises, but most exercise training is often overlooked. In this study, we searched for sports product patents and related literature that improve balance training and determined the direction of innovative design based on the different patent categories. Based on the results of the analysis, we have redesigned a model that can effectively provide innovative sports products with different angular misalignments and different displacements. In this research, we search, analyze and summarize the related patents and further identify the methods of innovative design to accomplish the required technologies for this design. The relevant design of this study is to use SolidWorks to draw 3D models for motion simulation and size checking. Then use the ANSYS11.0 command module to analyze the body structure of the force situation, to determine the strength and safety. Finally, make the original. Finally, this study hopes to provide a design reference for future sports product design engineers through this design process.
\end{abstract}

\section{Introduction}

The diminished sense of balance is one of the important indicators of the aging process. Normally healthy adults begin to exhibit initial signs of diminished balance after the age of 60 , and then degenerate more rapidly thereafter. The main reason is the degradation of multiple physiological functions, including the nervous system, skeletal joint system and cardiovascular system, all of which cause the degradation of "balance" function. And the degradation of balance will directly affect the daily life of the elderly, if combined with environmental factors (such as poor lighting, slippery ground) are often easy to make the elderly fall. The study found that $30 \%$ of elderly people over the age of 65 fell and one quarter of them were severely injured. About $5 \%$ of those who received medical examination had Fall twice or more. Research also shows that the chances of falling old people falling again are four times those of those who have never fallen. So preventing falls is very important to the elderly. Preventing falls can start with exercise, improving the home environment and using appropriate walking aids. The purpose of this study is to enhance the promotion of the balance of the elderly through training of balance exercises [1] [2] [3].

In general, after long-term practice, we can rely on the body's reflex and coordination ability to walk easily. However, modern people often get sedentary for a long time and cause muscle soreness for a long time. Even brain response and limbs balance Will be gradually reduced by the fitness equipment to maintain physical fitness, general exercise equipment by monotonous reciprocating exercise forging muscle endurance, and balance training machine can enhance the brain inner ear vestibular, semicircular canal and other organs and the coordination between the muscular Speed, suitable for promoting the brain development of young children, special education for people with intellectual disabilities, video game simulator and indoor surfing training and other purposes. This research by collecting relevant patents and existing products for analysis to find a simple and reliable mechanism with the lowest cost, To achieve the best training effect of the new balance training machine, so that both men and women can easily and safely experience the balance of exercise fun. These guidelines, written in the style of a submission to MATEC Web of Conferences, explain how to prepare your paper using Microsoft Word.

Please submit sources files directly to the conference organizer. If the conference editors chose to provide print-ready PDF documents to the publisher, you have to submit high-resolution PDF file with all fonts embedded (see PDF guidelines) instead of the sources files. In this case, please remember that no final corrections will be made by the publisher. There are quite a lot of patents and products available in balance training machines today. There are no motivation sources for "machine passive" and "motivation for machine". Please submit sources files directly to the conference organizer. If the conference editors chose to provide print-ready PDF documents to the publisher, you have to submit high-resolution PDF file with all fonts embedded (see PDF guidelines) instead of the sources files. In this case, please remember that no final corrections will be made by the publisher. There are 
quite a lot of patents and products available in balance training machines today. There are no motivation sources for "machine passive" and "motivation for machine".

\section{Patent classification and analysis}

There are quite a lot of patents and products available in balance training machines today. There are no motivation sources for "machine passive" and "motivation for machine". There are two major categories: machine passive balance training machine, the product itself is not Power-driven, rely on the user to move the body to stabilize the equipment, which has the advantages of small size, low cost, simple structure design is easy, often used to stimulate the development of vestibular vestibular training equipment, but the operation is too monotonous, long-term use of user fun gradually Lost, often can only be placed in the warehouse finally Section Headings.

The second type of active balance training machine has more than one actuator drive mechanism, which can make the pedal perform the deflection, bumpy and swivel as shown in Figure 1, and even the displacement in different angular directions, causing the user's body to disintegrate To do this, the muscle must be moved to maintain the balance of the body. To achieve this goal, an active balance training machine usually has multiple sets of original work units, large volumes and high costs. The following is a list of several active balance trainer patent agencies to introduce, from induction to find innovative design direction.

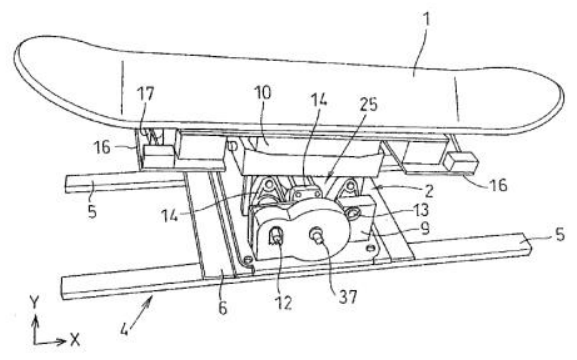

Figure 1. Pedal steering.

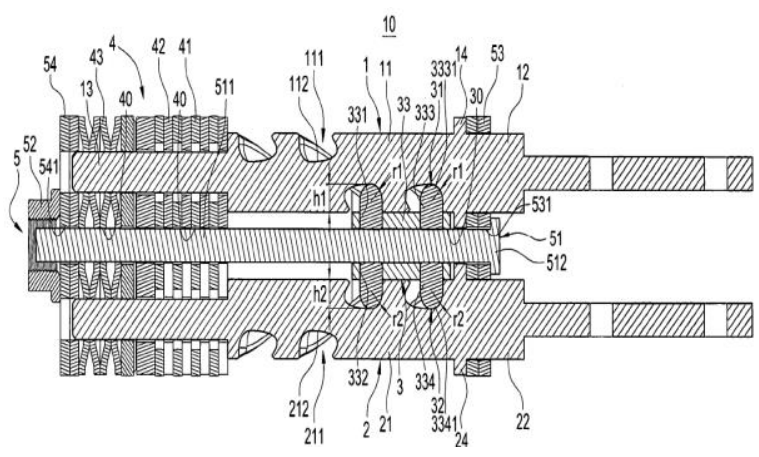

Figure 2. Twin Screw Drive Crank Slider.

Twin-screw-driven balance training machine [4], Figure 2 This patent is driven by the motor leadscrew, inverted crank slider mechanism slider, so that the crank angle changes, the use of two parallel linkage system to drive the pedal, This design can get the bumpy and the rotation of the two directions of the angle change, the disadvantage is unable to produce the user's horizontal displacement of the center of gravity, the use of screw drive can get accurate and stable output, but lost the speed of change, reducing user challenges. Please submit sources files directly to the conference organizer. If the conference editors chose to provide print-ready PDF documents to the publisher, you have to submit highresolution PDF file with all fonts embedded (see PDF guidelines) instead of the sources files. In this case, please remember that no final corrections will be made by the publisher [4].

As shown in Figure 3, the patent uses two sets of crank connecting pedals. Since the shafts of the two cranks are not parallel, it can generate complex 8-shaped swing and tilt and use a single motor via the bevel gear drives stable rotation of two sets of crankshafts and has a wide range of motion variation. Because of the simplified structure design, the cost is low and the manufacturing is easy. Only the direction of the displacement can not be controlled. Only the speed is adjusted, and the user feels closer to the vibration Body shaping sports equipment [5] [6] [7] [8].

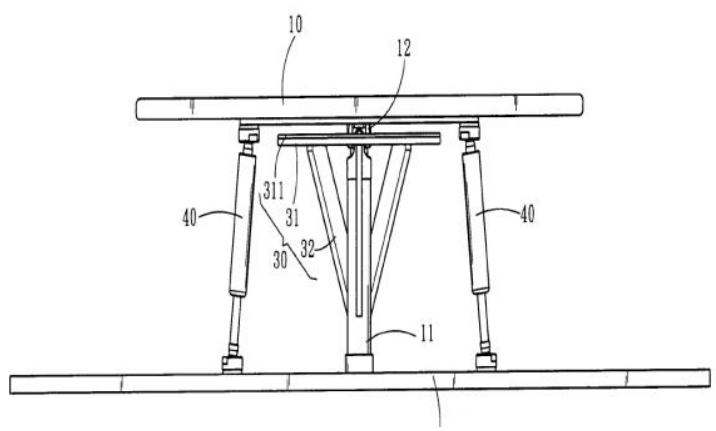

Figure 3. Double crank interlocking.

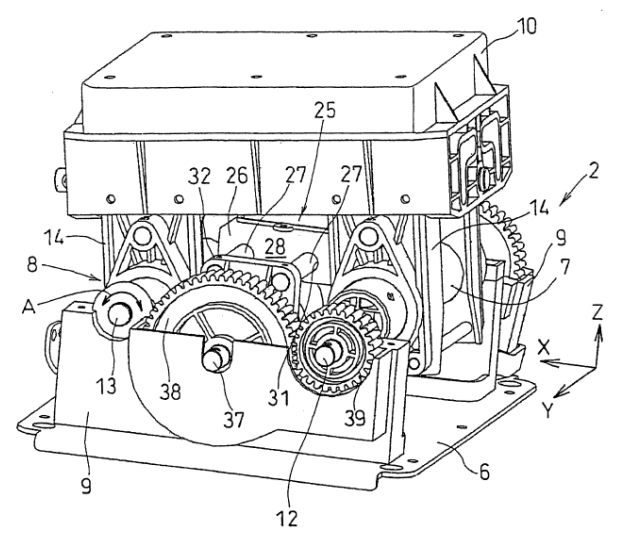

Figure 4. Composite Balance Trainer Driver.
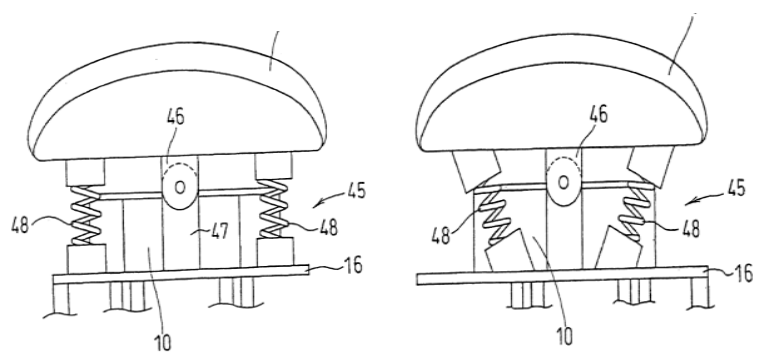

Figure 5. Composit balance follower. 


\section{The balance of training equipment design conditions}

The analysis of available patents and related products available after the hair

Now, the balance training machine designed in this study aims to train the indoor sports equipment that reflects the coordination between the body and the body, and provide the users of any age without any limitation of time and space to exercise and blood easily Cycle effect, so the definition of balance training machine basic design conditions, respectively, as follows:

(a) The design focus must be achieved with the least amount of parts, the ability to achieve the most movement, and the goal of reducing material costs.

(b) Is a surfing simulation training machine, machine running frequency is not high, but the pedal movement range and displacement range should be sufficient.

(c) Balance trainers need to provide better stability, so the lower the level, the better.

(d) It must be closer to real surfing and challenging.

(e) Designed to be safe and comfortable, to simplify and weight the components, and to have sufficient mechanical strength.

According to the above patent classification, "active" and "passive" balance trainers each have their own advantages and disadvantages. If one can design a sport capable of "active" and at the same time has a "passive" small volume for balance training Machine, I believe it will become a new consumer sports equipment.

\section{Mechanism design of balance training machine}

This time, we hope to achieve the most movement changing ability with the minimum number of machine parts. First of all, we should think about the mechanical structure that has a variety of rotation and displacement capabilities. The spherical joint is a single mechanical structure with yaw, pitch and roll rotation. However, To achieve rapid and accurate positioning, we must rely on an array of complex transmission mechanism to guide, do not meet the design conditions (a) described, so give up this idea; change to use six axes of rotation with two-axis rotation angle cylinder mechanism, partial Angle cylinder is composed of two cylinders combined with each other, shown in Figure 6, and the axis intersect to maintain a fixed angle, fixed one of the cylinder rotation, the other cylinder will show a reciprocating swing, and then use the ring into the swing cylinder to form a surface contact, the pedal Fixed on the ring, and the installation of the bracket to maintain the level to prevent the pedal group with the declination of cylindrical rotation, as long as the declination cylinder fixed axis, you can drive the pedal pitch, yaw, pitch ... reciprocating motion, to a single power input Resulting in biaxial angular displacement target, when the output axis and the drive shaft deflection angle of $10^{\circ}$, the motor per revolution The plate swings up and down while the output shaft produces $20^{\circ}\left( \pm 10^{\circ}\right)$ pitch, yaw angular displacement.

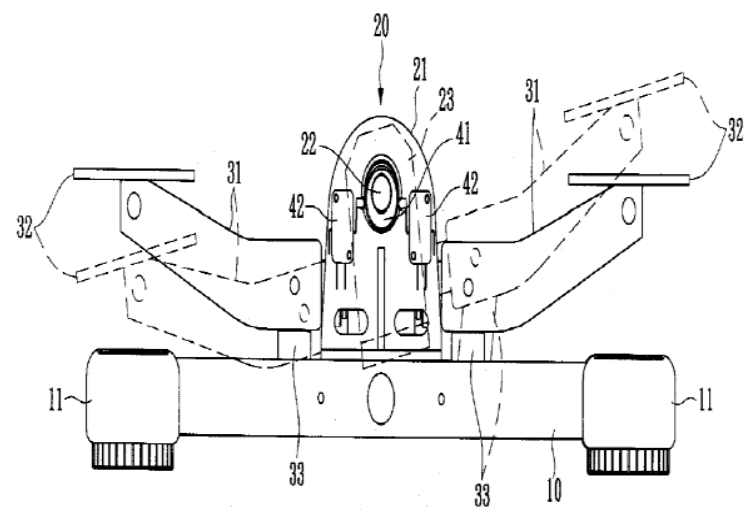

Figure 6. Angular cylinder.

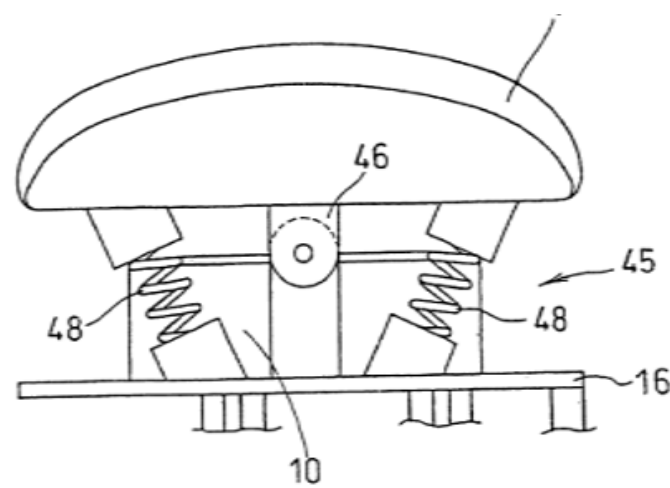

Figure 7. Pedal roll angle displacement front view.

If the pedal continuously changes in the stable angular displacement, it will soon lose the user's fun. Therefore, in combination with the passive balance training machine, the user must overcome the external force of the machine driven by the human body and the additional center of gravity from the passive balance training machine Change, and increase the use of the variability and challenges; Consider surfing the $\mathrm{Y}$ direction of the wave is less chance of encountering, so long as the surfer in the event of waves hit the pitch (Pitch), you can still stand on the water, stabilize the lateral (roll) can not fall into the water, so the passive balance training machine swing direction is set to rotate the $\mathrm{X}$-axis, the implementation of the method used to maintain the level of the bracket to remove, to change the angle cylinder drive shaft on both sides, each setting A set of compression springs, similar to the see-saw structure shown in Figure 7, is used to generate the roll angular displacement with a simple design, which meets the design conditions of Article (e) and reduces the size of the body to reduce manufacturing costs.

Horizontal displacement of the pedal includes $\triangle X$, $\triangle \mathrm{Y}$ biaxial translation and $\mathrm{Z}$ yaw, in order to obtain sufficient movement, use the horizontal position as shown in Figure 8 crank-slider mechanism as the base, the pedal is fixed on the even rod, by adjusting the length of the crank and the spindles, the appropriate pedal horizontal displacement is obtained, and the yaw from the crank is combined with the yaw angular displacement produced by the angling cylinder to produce more 
variability, You want to be more stable in the pedal above the more challenging.

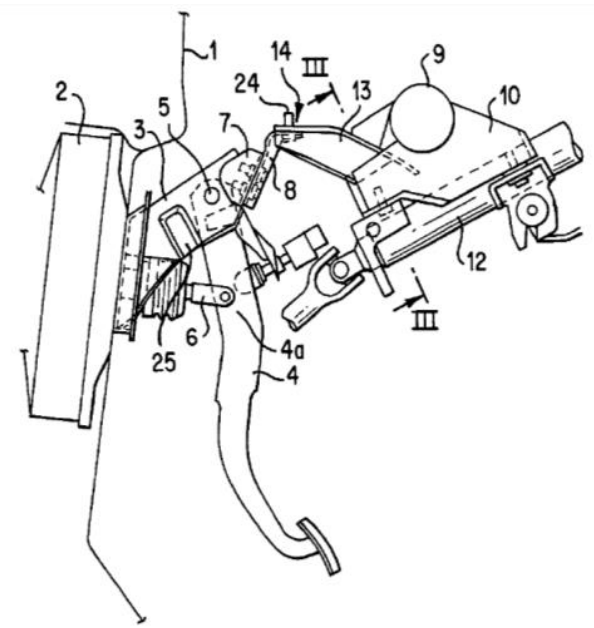

Figure 8. Pedal horizontal displacement mechanism.

\subsection{The Establishment of 3D Model and Stress Analysis of Balance Training Machine}

According to the design requirements to define the important size of the organization, the use of computeraided design software AutoCAD 2016 design, draw a detailed part portfolio diagram, and then further use SolidWorks2016 according to the size of 2D drawings to build the 3D model of Figure 9 and Figure 10 parts drawings and portfolio, Test whether the interference between the various components, and consider the ease of assembly, make reasonable modifications, and finally the use of computer graphics software for dynamic simulation of movement and horizontal displacement, you can show the range of components, and then design a sufficient size of the shell cover Internal agencies, to prevent the user due to improper use of the resulting risk of injury.

After completing the 3D model of the balance trainer and confirming that there is no interference on the part of the organization, the stress analysis of the structure is performed to ensure that the strength of the structural members and joints are within the safe range and the entity building can be avoided Finished to find the design strength of the defect. The materials used in the structural part of this study are based on structural steel A36 pipes of $1.2 \mathrm{~mm}$ thickness, while bolts are the material of medium carbon steel AISI1045. And simulate the stress analysis as shown in Figure 11, through the 120kg user's weight when operating on the balance trainer. The ANSYS software analysis shows that the maximum stress value is about $149.01 \mathrm{MPa}$, which occurs at the center of the base frame. The bolt material used in this study is AISI1045 medium carbon steel with a yield strength of $530 \mathrm{MPa}$. The structure is made of A36 structural steel. The yield strength of $250 \mathrm{MPa}$, so the bolt and the structure are not damaged, the safety factor of 1.68 , the strength of the solid structure within a certain range of safety.

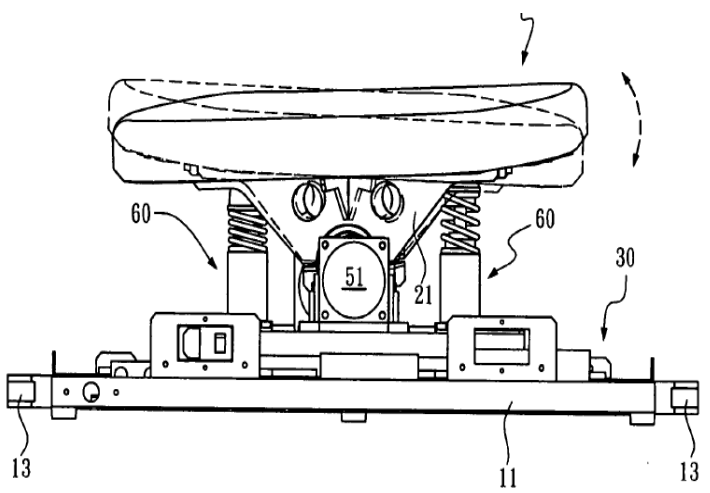

Figure 9. Passive roll rotation system.

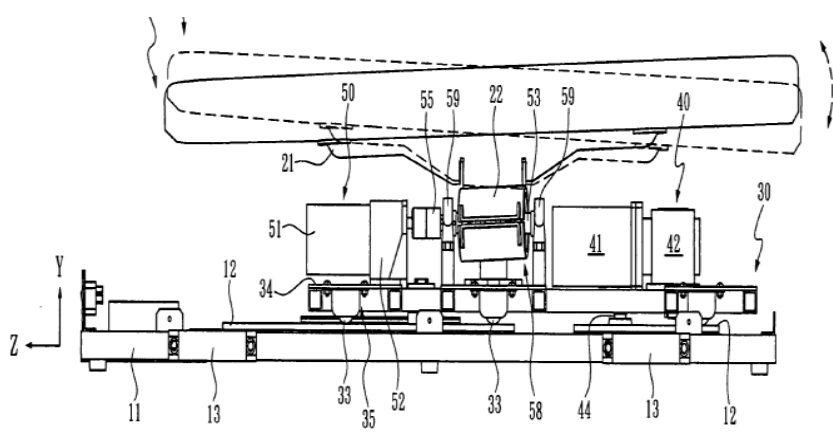

Figure 10. Intermediate base.

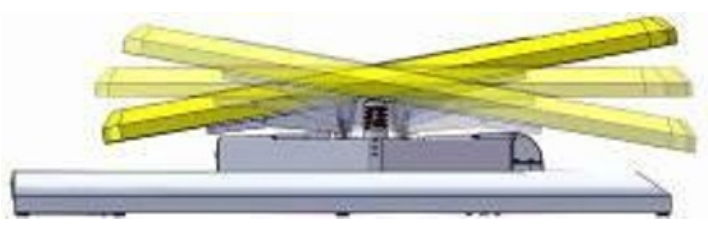

Figure 11. Simulation analysis.

\subsection{Prototyping}

After a series of complete design and analysis, the output drawings of all the parts were processed and manufactured, and some defects were partially improved. The prototype of Figure 12 and Figure 13 was assembled and the operation test was conducted to verify the original design concept. This design also places a sensor under the yaw mechanism and displays the balance of the user through the light on the display of Figure 13 to provide future balance training.

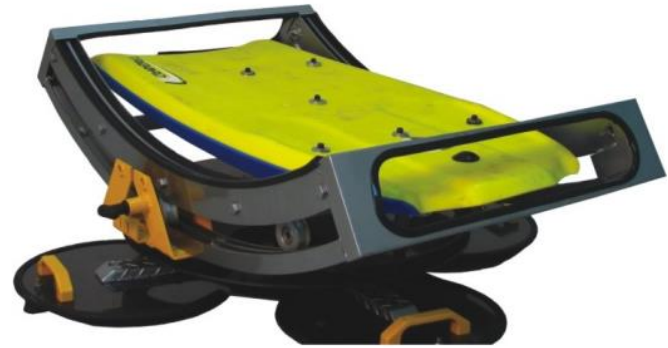

Figure 12. Prototype entity. 


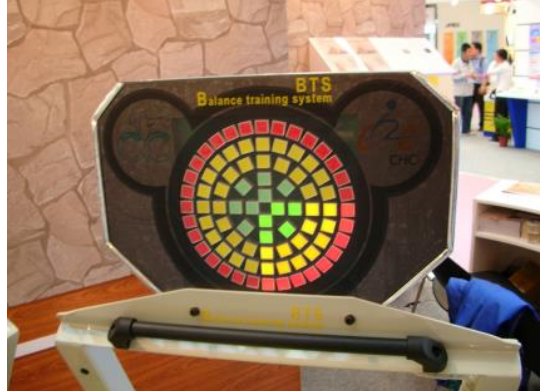

Figure 13. Bias correction sensing.

\section{Summary}

In this study, two DC motors were successfully used as the driving source to achieve the roll, pitch, and yaw three-axis rotation and balance exercise machine that balances the horizontal movement, and saves the parts cost by ingenious design without sacrificing sports performance. One of the directions for future development is to favor large-scale game simulation machines with a wider range of operation and more controllability. The other is the opposite, with light, thin, small size and more cost-saving home-balance training machines.

\section{References}

1. S.E. Syu, M.H. Hsu, Kunshan University of Science and Technology Institute of Mechanical Engineering Master thesis, Taiwan (2015)

2. Z. Shen, Jianguo University of Science and Technology Department of Automation Engineering and Electrical and Mechanical Systems Master thesis, Taiwan (2012)

3. Y.L. Chiu, J.N. Chen, Chaoyang University of Science and Technology Industrial Design Institute Master thesis, Taiwan( 2014)

4. R.P. Bittikofer, US patent announcement, Patent number: 5593380 (1997)

5. S. Ho, Republic of China patent announcement, Patent number : 310010 (2006)

6. Shimizu stretch one, Republic of China patent announcement, Patent number: 957555 (2006)

7. K. Grondys, I. Kott, A. Wiśniewska-Sałek, Polish Journal of Management Studies, 10(1), 45-53, 2014

8. S. Kot, Promet - Traffic - Traffico, 27 (5), 387-394. 2015 